\title{
An evaluation of palladium-based catalysts for the base-free borylation of alkenyl carboxylates
}

\author{
Gregory Gaube, Nahiane Pipaon Fernandez, and David C. Leitch* \\ Department of Chemistry, University of Victoria, 3800 Finnerty Rd. Victoria BC, CANADA, V8P 5C2. \\ *dcleitch@uvic.ca
}

Synthesis of organoboron derivatives is a key application of catalytic cross-coupling, with the Pd-catalyzed Miyaura borylation among the most versatile methods available. We have evaluated several Pd-based systems for borylation of alkenyl acetates and pivalates, with the optimal system heavily dependant on the substrate structure.

Boron-based functional groups serve as versatile synthetic handles in many metal-catalyzed cross-coupling reactions. ${ }^{1,2}$ As the Suzuki-Miyaura coupling has increased in importance over recent decades, so has the need for reactions that install the necessary boron-based functional groups. ${ }^{3}$ Following seminal work in this area by Miyaura, ${ }^{4} \mathrm{Pd}$-catalyzed borylation of halide or triflate electrophiles, typically with $\mathrm{B}_{2} \mathrm{pin}_{2}$ as the boron source and driven by the addition of a weak base, remains one of the most widely-used methods. ${ }^{5}$ Although there are numerous recent studies on improving this general reaction, 6,7 there remains a reliance on (pseudo)halide leaving groups, particularly bromides and triflates.

An alternative approach that can improve reaction mass efficiency and use new feedstocks/building blocks is the substitution of oxygen-based leaving groups via $\mathrm{C}-\mathrm{O}$ activation. ${ }^{8-12}$ Unfortunately, the requisite $\mathrm{C}-\mathrm{O}$ activation is difficult, leading to few systems reported for $\mathrm{C}-\mathrm{O}$ borylation using $\mathrm{Rh},{ }^{13,14} \mathrm{Ni}^{15-18}$ and more recently $\mathrm{Fe}^{19,20}$ (Figure 1a). Among the possible O-based electrophiles, aryl and alkenyl carboxylates offer several advantages, including ease of installation and base-free reaction conditions; ${ }^{21,22}$ however, there is only one report of successful borylation with this class of substrate using Rh catalysis. ${ }^{13}$ Recent work from our research group has shown that $\mathrm{Pd}(0)$ can undergo $\mathrm{C}-\mathrm{O}$ oxidative addition with heteroaryl and alkenyl carboxylates, and that Pd-catalyzed cross-coupling of alkenyl acetate and pivalate electrophiles with aryl boronic acids is possible under base-free conditions (Figure 1b). $21,23,24$ Here, we extend this reactivity to Pd-catalyzed borylation by evaluating three distinct catalytic systems using $\mathrm{Pd}(0)$ and $\mathrm{Pd}(\mathrm{II})$ precursors (Figure $1 \mathrm{c}$ ). a) Previous work: $\mathrm{Rh}, \mathrm{Ni}$, and $\mathrm{Fe}$ catalysis for $\mathrm{C}-\mathrm{O}$ borylation

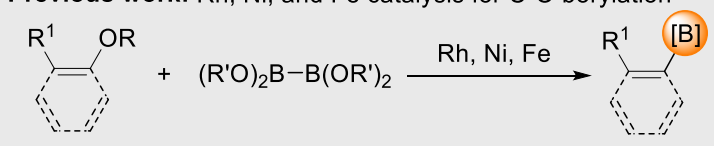

b) Pd-catalysis for $\mathrm{C}-\mathrm{O}$ activation: $\mathrm{Pd}(\mathrm{O}) /(\mathrm{II})$ or $\mathrm{Pd}(\mathrm{II})$ mechanisms

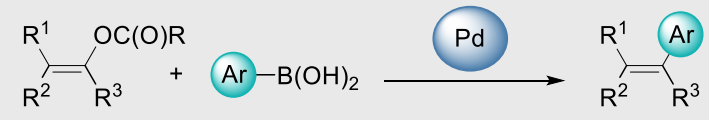

- easily accessible alkenyl carboxylates - air/moisture tolerant - commercially available boronic acids - base, oxidant, and halide-free - broad scope; heterocycles - room temperature reactivity

c) This work: Pd-catalyzed $\mathrm{C}-\mathrm{O}$ borylation

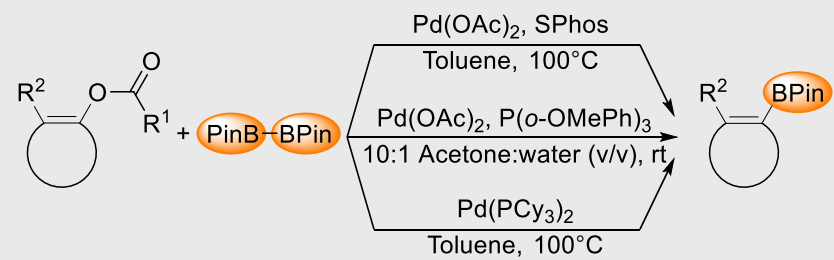

- 12 alkenyl carboxylate substrates evaluated with 3 catalyst systems - base-free borylation with both OAc and OPiv leaving groups

- reactivity enables further cross-coupling or net deoxygenation

Fig. 1 a) Prior catalytic approaches to $\mathrm{C}-\mathrm{O}$ borylation; b) $\mathrm{C}-\mathrm{O}$ activation in $\mathrm{C}-\mathrm{C}$ bond formation with arylboronic acids; $\mathrm{C}$ ) an evaluation of catalytic systems for bond formation with arylboronic acids
borylation involving $\mathrm{C}-\mathrm{O}$ activation

Prior screening on the Pd-catalyzed Suzuki coupling of alkenyl carboxylates revealed two suitable catalytic systems. One involves a mixture of $\mathrm{Pd}(\mathrm{OAc})_{2}$ and tris(orthomethoxyphenyl)phosphine to generate an active system in situ. This catalyst operates at room temperature under air and in the presence of water, likely through a Pd(II)-mediated mechanism. ${ }^{24-26}$ The second is $\mathrm{Pd}\left(\mathrm{PCy}_{3}\right)_{2}$, a single-component $\mathrm{Pd}(0)$ catalyst that is capable of $\mathrm{C}-\mathrm{O}$ oxidative addition at elevated temperatures. ${ }^{23}$ To determine if other $\mathrm{Pd} /$ ligand combinations could be active for borylation catalysis under base-free conditions, we conducted targeted microscale highthroughput screening of the reaction between $B_{2}$ pin 2 and dimedone-derived alkenyl acetate 1a (Figure 2). 

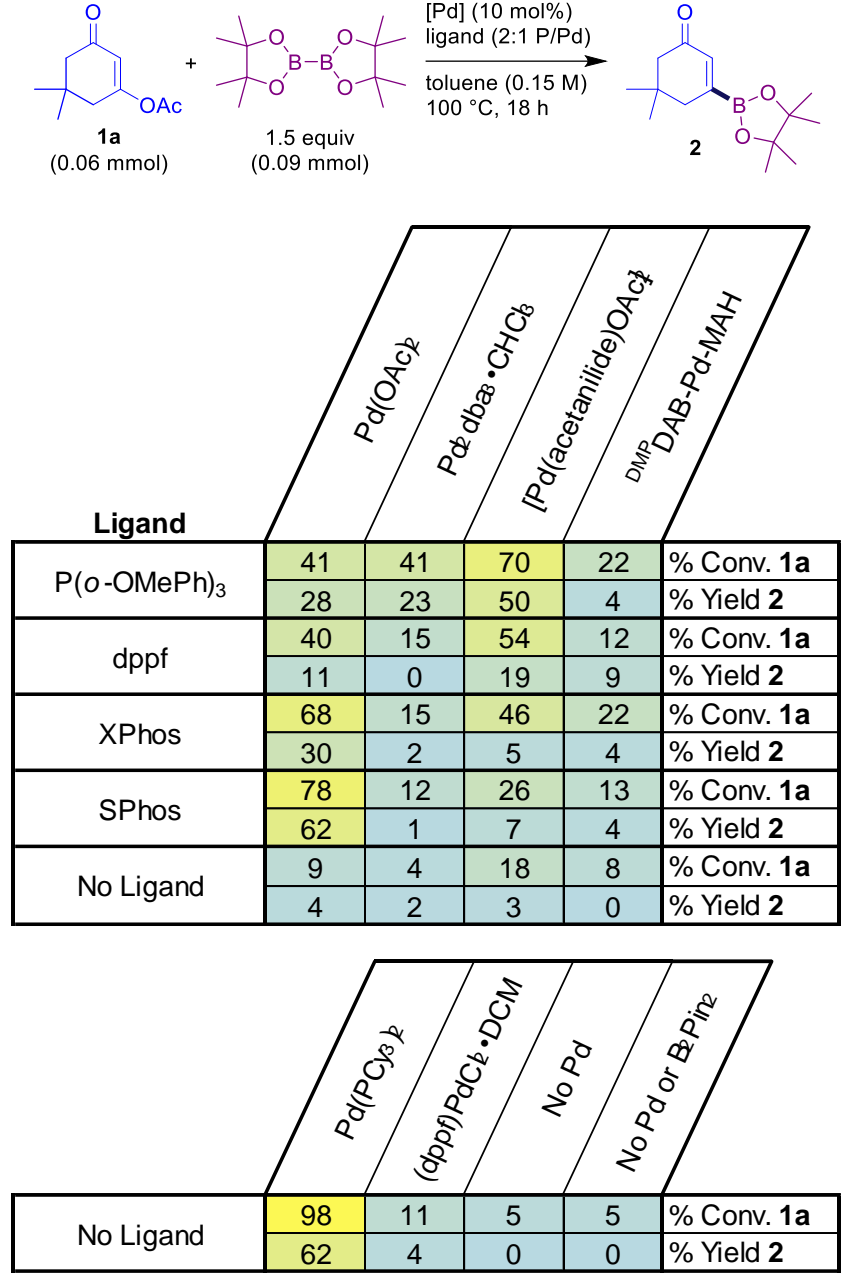

Fig. 2 Catalyst screening for the borylation of 1a. Conversion and yield determined by ${ }^{1} \mathrm{H}$ NMR spectroscopy using 1,3,5-trimethoxybenzene as an internal standard.

Initially we evaluated the efficacy of four ligands, including the previously identified $\mathrm{P}(\mathrm{o}-\mathrm{OMePh})_{3}$ and three effective crosscoupling ligands in dppf, XPhos, and SPhos. These were paired with four palladium sources: two $\mathrm{Pd}^{0}$ compounds including the recently reported DMPDAB-Pd-MAH, ${ }^{27}$ and two Pd" compounds. In addition, we evaluated two single-component precatalysts: $\mathrm{Pd}\left(\mathrm{PCy}_{3}\right)_{2}$ and (dppf) $\mathrm{PdCl}_{2}$. Several combinations enabled borylation to occur, with Pd" sources generally outperforming $\mathrm{Pd}^{0}$. Control experiments in the absence of ligand resulted in little to no product formation, and further control experiments with no $\mathrm{Pd}$ and with no $\mathrm{Pd}$ or $\mathrm{B}_{2} \mathrm{pin}_{2}$ resulted in little/no conversion of 1a. We identified the combination of $\mathrm{Pd}(\mathrm{OAc})_{2}$ and SPhos as the most active in situ candidate $(78 \%$ conversion and $62 \%$ solution yield) for further optimization. $\mathrm{Pd}\left(\mathrm{PCy}_{3}\right)_{2}$ is also effective, with a similar solution yield $(62 \%)$ though poorer mass balance.

Using the $\mathrm{Pd}(\mathrm{OAc})_{2} / \mathrm{SPhos}$ system as a starting point, a full factorial multivariate screen was designed to rapidly identify an optimal system (Figure 3 ). In addition to providing an improved, optimized set of conditions, this screen also offered additional insights into the catalytic system. Excess (3.0 equiv) SPhos reduced catalytic efficiency with both 4.0 and $10.0 \mathrm{~mol} \%$ $\mathrm{Pd}(\mathrm{OAc})_{2}$, consistent with a monophosphine-Pd active catalyst.
In all cases, $\mathbf{1 a}$ conversion and yield of $\mathbf{2}$ is improved with a large excess of $\mathrm{B}_{2} \mathrm{Pin}_{2}$. Using this excess generally increases the yield more than the conversion, leading to better overall mass balance. With $0.2 \mathrm{M}$ of $1 \mathrm{a}, 10 \mathrm{~mol} \% \mathrm{Pd}(\mathrm{OAc})_{2}, 15 \mathrm{~mol} \%$ SPhos and 2.0 equivalents of $\mathrm{B}_{2} \mathrm{Pin}_{2}$ an optimal yield was obtained.
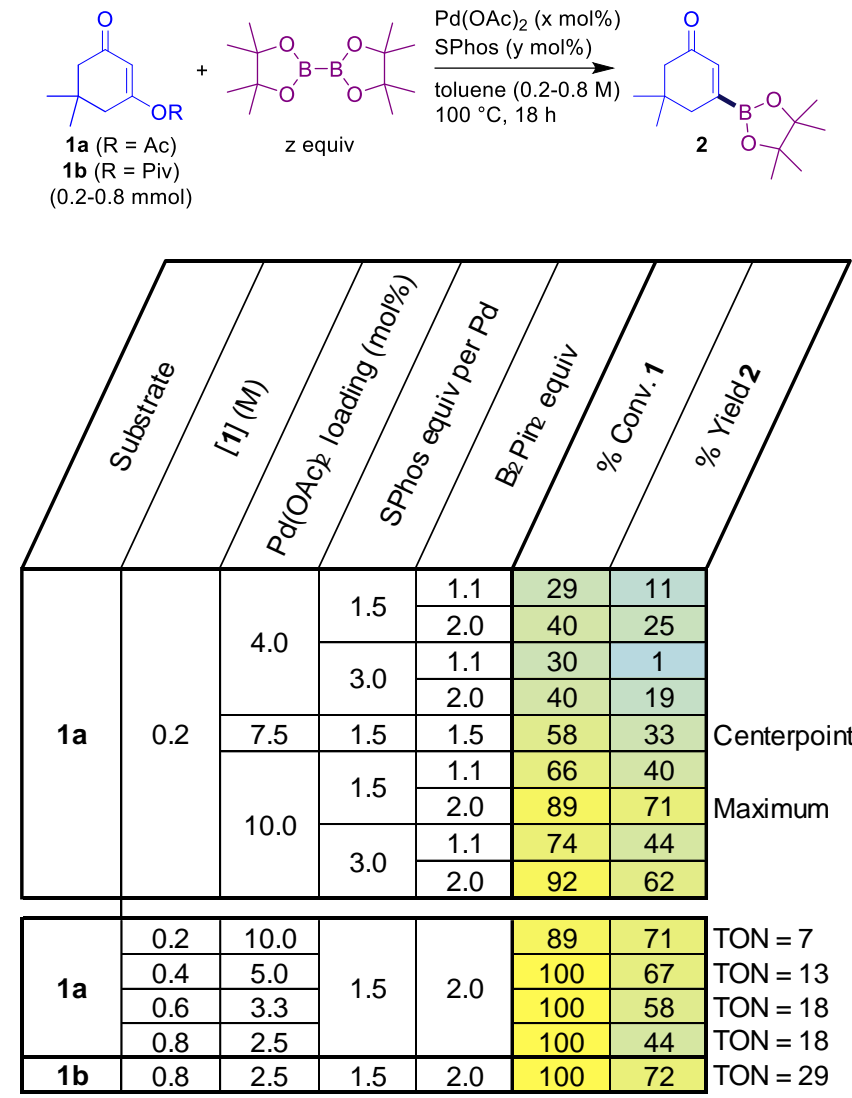

Fig. 3 Multivariate optimization of the borylation of 1a. Conversion and yield determined by ${ }^{1} \mathrm{H}$ NMR spectroscopy using 1,3,5-trimethoxybenzene as an internal standard.

While this set of conditions provides good solution yield of 2, it gives a very modest TON of 7; therefore, we sought to improve catalytic efficiency by increasing the substrate:catalyst ratio. From the full factorial screen, simply reducing $\mathrm{Pd}$ concentration has a deleterious effect on yield. Keeping the palladium and ligand concentration constant, we evaluated increasing substrate concentration from $[1 \mathrm{a}]=0.2 \mathrm{M}$ to $0.8 \mathrm{M}$, with a concomitant decrease in the substrate:catalyst mol ratio from $10 \mathrm{~mol} \% \mathrm{Pd}$ to $2.5 \mathrm{~mol} \% \mathrm{Pd}$. This does lead to increased TON; however, it also results in poorer mass balance, with the the chemical yield of $\mathbf{2}$ being reduced to only $44 \%$ at [1a] $=0.8$ M. We attributed this to competitive decomposition of 1a (e.g. by hydrolysis). To impede substrate decomposition, we assessed the more sterically-hindered and electron-rich pivalate derivative $\mathbf{1 b}$ at a concentration of $0.8 \mathrm{M}$. This results in significantly better mass balance and higher catalyst TON, with a $72 \%$ solution yield of $\mathbf{2}$. 


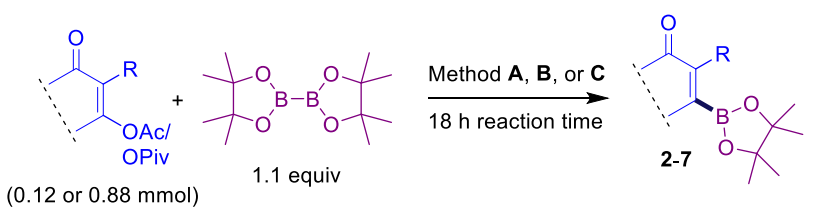

Method A: Pd(OAc) $)_{2}(4 \mathrm{~mol} \%) / \mathrm{P}(\mathrm{o}-\mathrm{OMePh})_{3}(6 \mathrm{~mol} \%)$, acetone/water $(10: 1,0.6 \mathrm{M})$, rt Method B: $\mathrm{Pd}(\mathrm{OAc})_{2}(2.5 \mathrm{~mol} \%) / \mathrm{SPhos}(3.8 \mathrm{~mol} \%)$, toluene $(0.8 \mathrm{M}), 100^{\circ} \mathrm{C}$ Method C: $\mathrm{Pd}\left(\mathrm{PCy}_{3}\right)_{2}(10 \mathrm{~mol} \%)$, toluene $(0.2 \mathrm{M}), 100^{\circ} \mathrm{C}$

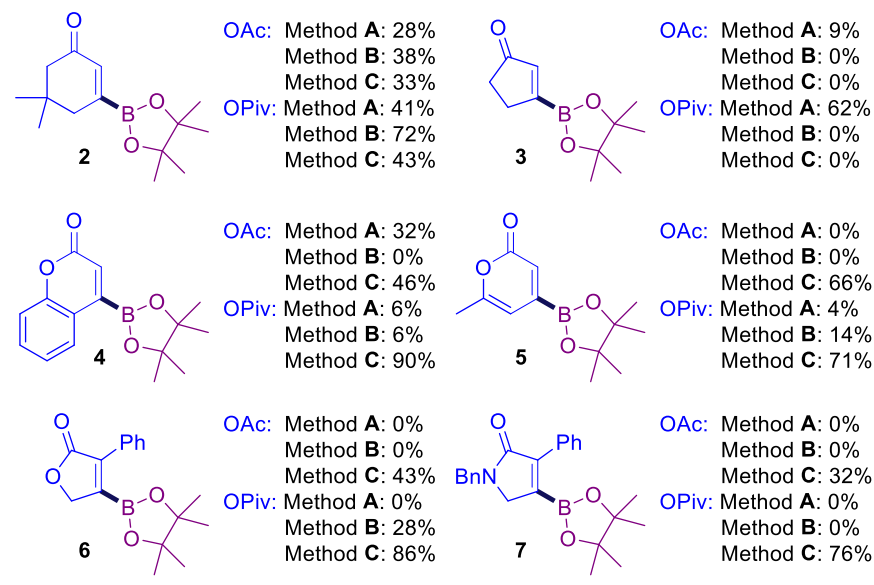

Fig. 4 Comparison of Pd-catalyzed methods for the borylation of alkenyl acetate and pivalate substrates. Substrate loading: $0.12 \mathrm{mmol}$ for Methods $\mathrm{A}$ and $\mathrm{C} ; 0.88$
$\mathrm{mmol}$ for Method $\mathrm{B}$. Yields determined by ${ }^{1} \mathrm{H}$ NMR spectroscopy using $1,3,5-$ trimethoxybenzene as an internal standard.

After this targeted optimization, we sought to evaluate the generality of this new protocol against the two previously developed systems for $\mathrm{C}-\mathrm{O}$ activation in Suzuki-type crosscoupling. We assembled a set of twelve alkenyl carboxylates with varying leaving groups (acetate and pivalate) and coumarin, pyrone, lactone, and lactam scaffolds as part of an exhaustive experimental design (Figure 4). Method A was previously used for the $\mathrm{Pd}(\mathrm{II})$-catalyzed $\mathrm{C}-\mathrm{O}$ activation crosscoupling, Method B is the optimized $\mathrm{Pd}(\mathrm{OAC})_{2} /$ SPhos system, and Method $\mathrm{C}$ was used for $\mathrm{Pd}(0)$-catalyzed $\mathrm{C}-\mathrm{O}$ activation cross-coupling. For the formation of $\mathbf{2}$, we observe the highest yield using Method B with the OPiv substrate, consistent with our multivariate optimization; however, Method B proved to be specific for this one system, failing to generate substantial product even for the structurally-related cyclopentanone $\mathbf{3}$. To form 3, only Method A with the OPiv substrate is effective, giving $62 \%$ solution yield.

Across these 36 reactions, several trends are apparent. Pivalate substrates are universally superior to the acetate substrates, which points to substrate decomposition as a potential issue in this chemistry. Method $\mathrm{C}$, involving $\mathrm{Pd}\left(\mathrm{PCy}_{3}\right)_{2}$ as a single-component precatalyst, is the most general, giving $>30 \%$ yield for every substrate other than the synthesis of $\mathbf{3}$. More broadly, these results reveal the importance of evaluating multiple methods for a given target substrate set: each of the three methods here performs best for different cases (Method A for 3, Method B for 2, and Method C for 4-7). Attempts to purify these compounds by chromatography or selective extractions led to either failure to remove pinacol-containing impurities, or decomposition of the product, so characterization was performed on crude reaction products.
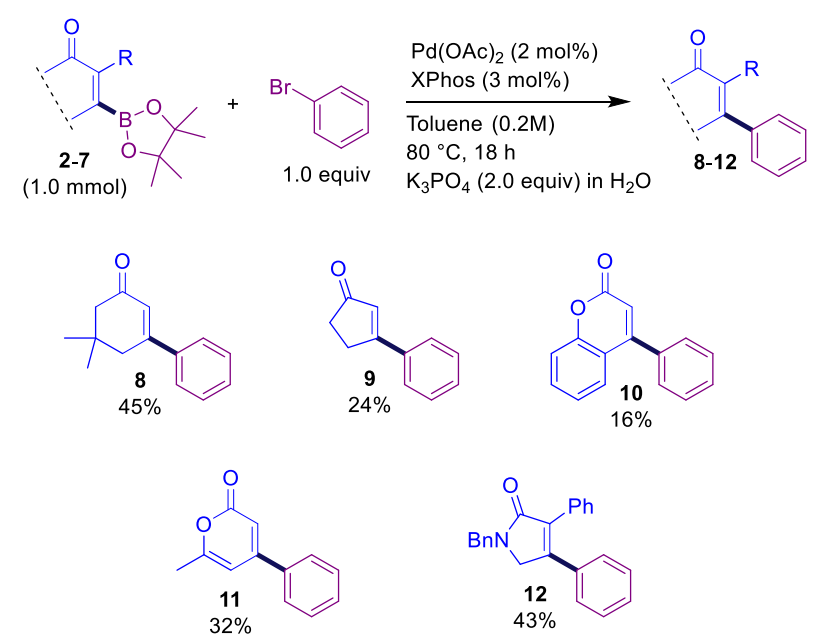

Fig. 5 Suzuki cross-coupling of prepared alkenyl boronates. Yields are for isolated compounds over two steps after column chromatography. Using boronate 6 gives conditions.

To assess these alkenyl boronates in cross-coupling, we performed preparative-scale Suzuki reactions. First, a $1 \mathrm{mmol}$ scale synthesis was conducted to form 2-7 using the optimal Method from Figure 4. During these investigations we discovered that even a mildly basic aqueous workup of these alkenyl boronates (saturated $\mathrm{NaHCO}_{3}$ ) prior to cross-coupling results in substantial protodeborylation. While this does provide a potentially simple two-step method for net deoxygenation of the $\beta$-dicarbonyl precursors (via acylation then Pd-catalyzed borylation/protodeborylation), it is problematic for realizing cross-coupling catalysis. We largely circumvented this issue by performing a neutral aqueous workup of 2-7, followed by a general and unoptimized Suzuki cross-coupling protocol (Figure 5). This enabled the isolation of the arylated products $\mathbf{8 - 1 2}$, despite the observed instability of the boronates; boronate 6 did prove to be too unstable toward protodeborylation for cross-coupling to be successful.

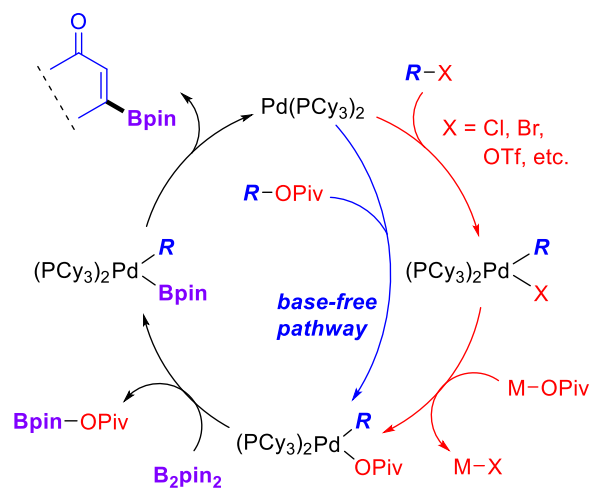

Fig. 6 Catalytic mechanism for established Miyaura borylation (red) and $\mathrm{C}-\mathrm{O}$ activation borylation (blue). The incorporation of the carboxylate into the therefore base-free borylation.

With respect to mechanism, we propose that reactions involving Method $C$ undergo sequential oxidative addition of the $\mathrm{C}-\mathrm{O}$ bond, followed by direct transmetallation between the resulting $\mathrm{Pd}(\mathrm{II})$ carboxylate and $\mathrm{B}_{2} \mathrm{pin}_{2}$ (Figure 6). This contrasts with standard Miyaura borylation in that base is not required 
for the salt metathesis reaction to replace the (pseudo)halide after initial oxidative addition. Notably, we observe no homocoupling byproducts during borylation resulting from a tandem borylation/Suzuki sequence, wherein the product RBpin would undergo direct transmetallation with the palladium(II) carboxylate intermediate. This is in contrast to previous observations from our laboratory where rapid transmetallation/reductive elimination is observed with $\mathrm{PhB}(\mathrm{OH})_{2} .^{23}$

\section{Conclusions}

Through a targeted multivariate optimization and an extensive parallel evaluation of conditions and substrates, we have demonstrated the base-free, Pd-catalyzed borylation of alkenyl carboxylates. Depending on the nature of the substrate, different catalyst/solvent combinations are most effective, with alkenyl pivalates outperforming their acetate counterparts. The resulting alkenyl boronates have varying stability with respect to protodeboronation, but still undergo Suzuki cross-coupling. This base-free borylation is enabled by the direct oxidative addition of the substrate to $\mathrm{Pd}(0)$, giving a $\mathrm{Pd}(\mathrm{II})$ carboxylate that can undergo direct transmetallation.

\section{Conflicts of interest}

There are no conflicts to declare.

\section{Acknowledgements}

We acknowledge with respect the Lekwungen peoples on whose traditional territory the University of Victoria (UVic) stands, and the Songhees, Esquimalt and WSÁNEĆ peoples whose historical relationships with the land continue to this day. The authors thank UVic, NSERC, CFI, BCKDF, and MITACS (RTA to GG) for funding this work.

\section{Notes and references}

1 J. Magano and J. R. Dunetz, Chem. Rev., 2011, 111, 2177-2250.

2 E. C. Neeve, S. J. Geier, I. A. I. Mkhalid, S. A. Westcott and T. B. Marder, Chem. Rev., 2016, 116, 9091-9161.

3 D. G. Brown and J. Boström, J. Med. Chem., 2016, 59, 4443-4458.

4 T. Ishiyama, M. Murata and N. Miyaura, J. Org. Chem., 1995, 60, 7508-7510.

5 T. Ishiyama and N. Miyaura, J. Organomet. Chem., 2000, 611, 392-402.

6 M. Wang and Z. Shi, Chem. Rev., 2020, 120, 7348-7398.

7 S. Barroso, M. Joksch, P. Puylaert, S. Tin, S. J. Bell, L. Donnellan, S. Duguid, C. Muir, P. Zhao, V. Farina, D. N. Tran and J. G. de Vries, J. Org. Chem., 2021, 86, 103-109.

8 D.-G. Yu, B.-J. Li and Z.-J. Shi, Acc. Chem. Res., 2010, 43, 14861495.

9 B. M. Rosen, K. W. Quasdorf, D. A. Wilson, N. Zhang, A.-M. Resmerita, N. K. Garg and V. Percec, Chem. Rev., 2011, 111, 13461416.

10 B.-J. Li, D.-G. Yu, C.-L. Sun and Z.-J. Shi, Chem. Eur. J., 2011, 17, 1728-1759.
11 M. Tobisu and N. Chatani, Top. Curr. Chem., 2016, 374, 41.

12 Z. Qiu and C.-J. Li, Chem. Rev., 2020, 120, 10454-10515.

13 H. Kinuta, J. Hasegawa, M. Tobisu and N. Chatani, Chem. Lett., 2014, 44, 366-368.

14 H. Kinuta, M. Tobisu and N. Chatani, J. Am. Chem. Soc., 2015, 137, 1593-1600.

15 K. Huang, D.-G. Yu, S.-F. Zheng, Z.-H. Wu and Z.-J. Shi, Chem. Eur. J., 2011, 17, 786-791.

16 C. Zarate, R. Manzano and R. Martin, J. Am. Chem. Soc., 2015, 137, 6754-6757.

17 K. Nakamura, M. Tobisu and N. Chatani, Org. Lett., 2015, 17, 6142-6145.

18 M. Tobisu, J. Zhao, H. Kinuta, T. Furukawa, T. Igarashi and N. Chatani, Adv. Synth. Catal., 2016, 358, 2417-2421.

19 X. Zeng, Y. Zhang, Z. Liu, S. Geng, Y. He and Z. Feng, Org. Lett., 2020, 22, 2950-2955.

20 S. Geng, J. Zhang, S. Chen, Z. Liu, X. Zeng, Y. He and Z. Feng, Org. Lett., 2020, 22, 5582-5588.

21 J. Becica and D. C. Leitch, Synlett, 2021, 32, 641-646.

22 G. Toupalas and B. Morandi, ChemRxiv, 2021, DOI:10.26434/chemrxiv.14217314.v1.

23 J. Becica, G. Gaube, W. A. Sabbers and D. C. Leitch, Dalton Trans., 2020, 49, 16067-16071.

24 J. Becica, O. R. J. Heath, C. H. M. Zheng and D. C. Leitch, Angew. Chem. Int. Ed., 2020, 59, 17277-17281.

25 S. E. Shockley, J. C. Holder and B. M. Stoltz, Org. Process Res. Dev., 2015, 19, 974-981.

26 A.-L. Lee, Org. Biomol. Chem., 2016, 14, 5357-5366.

27 J. Huang, M. Isaac, R. Watt, J. Becica, E. Dennis, M. I. Saidaminov, W. A. Sabbers and D. C. Leitch, ACS Catal., 2021, 11, 5636-5646. 